\title{
El emotivismo y su influencia en las teorías contemporáneas del desarrollo moral
}

\author{
Emotivism and its influence on \\ contemporary theories of moral development
}

\begin{abstract}
Resumen
Durante años, filósofos y científicos interesados en el tema de la moral han intentado establecer la influencia de las emociones sobre nuestros juicios acerca de lo moralmente adecuado o inadecuado. Mientras muchos consideran la emoción como un obstáculo para este propósito, sugiriendo que la finalidad de la moral implica un dominio de la razón sobre los afectos, otros han encontrado en ella el fundamento para comprender nuestra vida moral. Esta postura que defiende que el significado de "bueno o malo" depende de nuestros sentimientos o actitudes es denominada emotivismo moral. En el siguiente documento se presenta una reflexión en torno al emotivismo moral como doctrina en la filosofía moral y su influencia en las posturas científicas contemporáneas en la psicología del desarrollo moral.
\end{abstract}

\begin{abstract}
For years philosophers and scientists interested in the theme of morals have tried to establish the influence of emotions on our judgments of what is morally correct or incorrect. While many consider emotion as an obstacle to this purpose, suggesting that the end purpose of moralism implies the domination of reason over affection, others have found in it the basis for understanding our moral life. This position, which argues that the meaning of "good or evil" depends on our feelings or attitudes is called "moral emotivism". The following document presents a reflection on moral emotivism as a doctrine in moral philosophy and its influence on contemporary positions in the psychology of moral development.
\end{abstract}

Keywords: moral, emotion, emotivism, moral development

Palabras clave: moral, emoción, emotivismo, desarrollo moral.

Recibido el 28 de mayo de 2012 y aprobado el 28 de junio de 2012.

1 Juan Carlos Marulanda es Psicólogo de la Universidad Nacional, docente del programa de Psicología de Uniminuto.

Polisemia No. 13, 76 - 85. El emotivismo y su influencia en las teorías contemporáneas del desarrollo moral. Bogotá, ISSN: 1900-4648. Enero - junio de 2012 
L a moral ha ocupado un lugar importante como tema de reflexión en la historia del pensamiento occidental. La pregunta por "cómo debemos vivir", interrogante sobre el que tanto discurrió la filosofía socrática, se replica una y otra vez en las diferentes escuelas y autores edificadores de la cultura de nuestra época. Sin embargo, y a pesar de la diversidad de teorías y propuestas que intentan responder al problema de la moral, y de los repetidos intentos por elaborar un sistema comprensivo de principios que puedan ser rectores de las acciones humanas, el panorama se muestra aún nebuloso y problemático; cada teoría moral esgrime argumentos que procuran defender una concepción sobre la vida moral y, a su vez, atacar y demostrar los errores contenidos en las propuestas rivales.

La tradición de la discusión en torno a la moral se ha desarrollado sobre la base de tres concepciones distintas; éstas representan el tallo del cual se desprenden las diversas perspectivas que han entrado en conflicto. Una primera concepción surgió en el contexto político de la antigua Grecia; se instauró gracias al desarrollo del pensamiento socrático y perduró hasta el final de la Edad Media. Defiende una idea de moral basada en la virtud y las prácticas sociales. La postura aristotélica frente a la ética es quizás la más elaborada y la que más ha logrado influencia en el mundo occidental.

Las otras dos posturas encuentran como antecedentes las corrientes filosóficas posterio- res a la Edad Media y se forjan en el seno de la Ilustración. Una de ellas, crítica de la tradición heredada de los pensadores socráticos, fundamentalmente de la ética aristotélica, fue defendida por la corriente empirista que opuso las pasiones y las emociones humanas a la virtud y la razón como fundamento para la vida moral. Resaltan dos teorías: de una parte, la visión de la moral edificada sobre el egoísmo y el temor, defendida por Hobbes; de otra parte, la teoría de Hume, que ubica el fundamento de la moral en los afectos y sentimientos.

La otra es la postura inaugurada por Kant. Su idea del imperativo categórico como criterio para la conformación de principios morales defiende el papel de la razón como la base de todo juicio y acción moral. Para este autor, el problema moral estaba en lograr, a través del intelecto, establecer principios universales que guiaran nuestras acciones, independientes de las condiciones particulares de una situación. Su sistema se convirtió en el fundamento de las teorías liberales modernas acerca de la ética y la justicia.

Este debate filosófico acerca de la moral ha gozado de una acogida significativa dentro de la psicología del desarrollo. Dentro de este campo son múltiples y novedosas las investigaciones y teorías en torno al tema. No obstante, los hallazgos y conclusiones expuestos por diversos autores tan sólo se suman a las contradicciones presentadas en el escenario filosófico.

Es así como la psicología del desarrollo moral constituye un campo disgregado, en donde 
conviven diversas visiones rivales que discuten sobre la forma en que debe entenderse lo moral y que intentan explicar el comportamiento prosocial, centrándose en una sola dimensión. Las teorías cognitivas de Kohlberg y Piaget se han centrado en el problema del razonamiento moral y explican el desarrollo moral desde una perspectiva estructuralista (Yáñez, Perdomo \& Mojica, s/f). Las teorías basadas en el aprendizaje se han fijado en el comportamiento de cooperación y su relación con conceptos como refuerzo, castigo e imitación. Finalmente, se encuentran las teorías del desarrollo emocional y motivacional que destacan el papel de las llamadas emociones prosociales como motor de todo comportamiento moral.

Con respecto a este último grupo de teorías, llama la atención las recientes investigaciones en torno a la empatía y su importancia para el desarrollo moral. A partir de estos trabajos se ha logrado consolidar evidencia suficiente en favor del vínculo entre los estados emocionales y las acciones y juicios morales. Los aspectos conductuales y cognitivos, abordados por otras teorías en el campo del desarrollo moral, pasan a ser re-explicados bajo esta mirada socio afectiva de la dimensión moral.

El propósito del siguiente texto es exponer la discusión filosofía en torno a la emoción como fundamento de la moral, discusión de la cual surge el concepto de empatía que ocupa un lugar central en las teorías emocionales y motivacionales del desarrollo moral en la psicología contemporánea. En segundo lugar, exponemos la manera en que es explicada la dimensión moral a partir de las concepciones heredadas de dicha discusión filosófica.

\section{El argumento emotivista en la filosofía moral}

Más allá de un intento de explicación del desarrollo de la moral, o más bien, de nuestra capacidad para actuar según las proscripciones dictaminadas por las normas morales, la teoría emocional retoma y se muestra partidaria de una de las posturas más importantes en la historia de la filosofía moral. Me refiero al llamado emotivismo moral, defendido en un comienzo por la corriente empirista y retomada luego por teorías éticas como el utilitarismo y sus versiones actuales.

El emotivismo puede resumirse en la idea de que un juicio moral es tan solo la expresión de una emoción, un sentimiento, una intención o una norma del hablante (Harman, 1996). En palabras de MacIntyre, el emotivismo moral es:

la doctrina según la cual los juicios de valor, y más específicamente los juicios morales, no son nada más que expresiones de preferencias, expresiones de actitudes o sentimientos, en la medida en que estos poseen un carácter moral o valorativo (2001, p. 26).

De esta manera, no es posible establecer el carácter de verdad o falsedad de los juicios morales, porque el significado de estos juicios se encuentra sujeto a los sentimientos que despiertan en cada persona. Podemos considerar esta característica como un argumento en favor del relativismo moral.

La postura emotivista moderna representa una tradición inaugurada en la filosofía política de Thomas Hobbes. El pensamiento de Hobbes se presenta como una reformulación radical de las concepciones tradicionales del modo de vida de los hombres basado en la virtud defendido por la filosofía clásica y la escolástica medieval. Para este autor, su filosofía se edifica sobre la base de un conocimiento científico acerca de la naturaleza humana (Strauss \& Cropsey, 1993). Esto lo convierte en uno de los primeros autores que intentaría edificar una teoría moral sobre una base científica.

Hobbes alude a conceptos como el placer y las pasiones para explicar la naturaleza de la moralidad. Hablar de las emociones o las pasiones humanas como fundamento de la moral es apelar, desde algunos puntos de vista, a una solución que evade con astucia todo conjunto de razonamientos y conceptos complicados a 
los que se había acostumbrado buena parte de la filosofía occidental y que fueron rechazados posteriormente por las escuelas empiristas del siglo XVIII y el positivismo científico (Strauss \& Cropsey, 1996).

El emotivismo de Hobbes parte de su admiración hacia la actividad científica que comenzaba a desarrollarse en su época. La conducta humana debía interpretarse en función de la psicología mecanicista de las pasiones, refiriéndose a estas últimas como la fuerza que empuja al hombre desde atrás. Así lo señala en un pasaje de su obra Leviatan:

Lo que de algún modo es objeto de cualquier apetito o deseo humano es lo que con respecto a él se llama bueno. Y el objeto de su odio o aversión, malo; y de su desprecio, vil e considerable o indigno. Pero estas palabras de bueno, malo y despreciable siempre se usan en relación con la persona que las utiliza. No son siempre y absolutamente tales, ni ninguna regla de bien y de mal puede tomarse de la naturaleza de los objetos mismos, sino del individuo (donde no existe Estado) o (en un Estado) de la persona que lo representa; o de un árbitro o juez a quien los hombres permiten establecer e imponer como sentencia su regla del bien y del mal (2010, p. 46).

Es evidente que para Hobbes lo bueno o lo malo, aquello que guía la conducta, está determinado por las pasiones y aversiones de cada cual. Cuando un hombre dice que algo es bueno, está mostrando simplemente que le agrada. Las pasiones dan lugar a las acciones, y los hombres son guiados por sus opiniones y su imaginación acerca de lo bueno y lo malo. La razón no somete las pasiones, tan sólo facilita el camino hacia lo que el hombre desea.

No obstante, Hobbes va más allá en la búsqueda de una teoría moral sobre la base del entendimiento de la naturaleza humana. Hobbes busca en realidad justificar la existencia de un soberano, el Estado, regulador de la relaciones entre los hombres. Su búsqueda lo lleva a la idea de un estado pre político o estado de naturaleza en donde los hombres se encuentran en constante pugna. Los hombres están en guerra por- que cada uno sigue lo que su naturaleza le dicta, que es la búsqueda de su propia conservación. Entonces, el hombre es egoísta por naturaleza, en tanto actúa buscando su propio bien incluso a expensas del daño a otros. Pero así como el hombre ve la posibilidad de dañar a otros para lograr su beneficio, siente también temor de ser agredido. Ese temor lo conduce a la búsqueda de un contrato en dónde cede su poder, el de actuar en contra de otros, a cambio de que los otros cedan también su poder y así se vea resguardado de cualquier daño. El contrato da origen al Estado, que es el soberano y el único que posee el poder de dañar a otros, ejerciendo ese poder con el propósito que nadie incumpla el contrato pactado (Strauss \& Cropsey, 1996; McIntyre, 2006).

La fuerza del Estado permite entonces ejercer el derecho a la conservación de la vida. De esta forma, Hobbes concibe una moral considerada como el instinto pacífico inspirado por el temor. Entonces, la ley moral o la ley natural se conciben como si procedieran del derecho natural, que es el derecho a la propia conservación; el hecho moral constituye un derecho, no un deber (Strauss \& Cropsey, 1996). En resumen, el hombre que Hobbes describe actúa conforme a las normas llevado por el temor, el egoísmo y el interés. No existe nada distinto a las pasiones humanas que permita explicar la moral.

Casi un siglo después, David Hume plantea una versión del emotivismo moral mucho más elaborada y radical que la que encontramos en Hobbes. En el Tratado de la Naturaleza Humana (2005), Hume expone una diversidad de argumentos conducentes a negar la posibilidad de que aquello que llamamos moral emerja del ejercicio de la razón. Su postura parte de la definición de la razón como una esclava de las pasiones humanas. Las acciones son fruto del placer o la aversión hacia los objetos y, en ese sentido, la razón sólo puede informar a la voluntad acerca de la relación de esos objetos y de las vías para conducirnos a ellos, más no de su placer o aversión. Así, para Hume la cognición 
moral no puede separarse de nuestra propensión o aversión a la acción, o de otra forma, no se puede saber lo que es moralmente bueno sin sentir una inclinación hacia ello.

Para Hume, la moral tiene que ver con aquello que es obligatorio, lo que se debe hacer. Aquello que resulta obligatorio sólo se puede conocer a través de la experiencia, por lo cual no puede emerger de la razón. Dado que sólo la pasión puede influir en la voluntad para otra acción, podemos considerar que son los afectos morales el fundamento de la moral (Strauss \& Cropsey, 1996; Schneewind, 2009). De esta manera, Hume considera que la distinción entre la virtud y el vicio depende más bien de los que llama un sentimiento moral. En la tercera parte del tratado lo expone así:

La moralidad, por consiguiente, es más propiamente sentida que juzgada, aunque este sentimiento o afección es comúnmente tan suave y sutil que nos inclinamos a confundirlo con una idea, según nuestra costumbre de tomar unas cosas por otras cuando existe entre ellas una gran semejanza (2005, p. 397).

La virtud, concebida por Hume, no es aprobada por ser virtud, sino que es virtud por el hecho de ser aprobada. La impresión que surge de la virtud es placentera, mientras que la que surge del vicio es desagradable. No significa que la aprobación sea un inferencia del placer, sino que la aprobación es reducida al placer. Por lo cual, los sentimientos morales no son más que placer y dolor. Pero son placer y dolor de una clase particular, en la manera en que son experimentados de manera diferentes a otros. Estas particularidades son: (1) sólo surgen de la consideración y el carácter de los seres racionales; (2) surgen cuando éstas son consideraciones sin referencia a nuestro interés particular (Strauss \& Cropsey, 1996).

A diferencia de Hobbes, Hume rechaza la idea de una moral egoísta, dicho de otra forma, la concepción de que las normas morales surgen del egoísmo y la competencia humana en el estado de naturaleza. Pero a la vez, Hume evita afir- mar que exista una benevolencia general entre los hombres. De no existir la competencia y el egoísmo, no sería necesaria la justicia, entendida por Hume como una virtud artificial creada para el bien de la sociedad.

Para rechazar la teoría del egoísmo y a la vez escapar de la idea de una benevolencia generalizada, Hume acude al concepto de simpatía, sentimiento que permite hacer propios los placeres y dolores de otros de una manera mecánica, sin que haya una buena voluntad o un sentimiento egoísta hacia los demás. Hume explica en el tratado la simpatía de la siguiente manera:

Los espíritus de los hombres son similares en sus sentimientos y operaciones, y no puede ser influido uno de ellos por alguna afección de la que todos los demás no sean en algún grado susceptibles. Lo mismo que en las cuerdas enlazadas, de un modo igual, el movimiento de la uno se comunica al resto de ellas, las afecciones pasan rápidamente de una persona a otra y ejecutan movimientos correspondientes a toda criatura humana. Cuando yo veo los afectos de la pasión en la voz y los gestos de una persona mi espíritu pasa inmediatamente de estos afectos a sus causas y se forma una idea vivaz de la pasión tal, que se convierte en el momento en la pasión misma. De igual modo, cuando yo percibo las causas de una emoción mi espíritu es llevado a sus efectos y afectada con una emoción igual. [...] Ninguna pasión de otro sujeto se descubre por si misma inmediatamente al espíritu. Solamente somos sensibles a sus causas y efectos. De esto inferimos la pasión y, por consecuencia, éstos son los que dan lugar a nuestra simpatía (2005, p. 480).

Lo que Hume revela es su creencia en la existencia de un sentimiento natural en los hombres que los lleva a actuar con benevolencia o según las virtudes naturales. Sin embargo, no es un sentimiento que se extienda a todos los seres humanos, sino tan sólo a aquellos con los que guardamos algún grado de cercanía o familiaridad. Es por esta razón que la justicia se hace necesaria, a saber, aparece allí donde la simpatía no se extiende.

La teoría emotivista cobra una nueva forma en la filosofía contemporánea con Charles 
L. Stevenson (Rachels, 2008 y McIntyre, 2001). Para Stevenson, el lenguaje moral, a diferencia del lenguaje común que describe hechos, funciona como un medio para influir sobre la conducta de los demás. Por ejemplo, la proposición: "esto es bueno" quiere decir lo mismo que: "yo apruebo esto, hazlo tú también", mostrando así que el juicio moral señala una actitud y, al mismo tiempo, intenta influir en la acción de otra persona (McIntyre, 2001). Podemos entender, pues, esta versión modificada del emotivismo moral como una teoría general del significado de los conceptos morales, ligado al uso que tienen dichos juicios en el entorno social.

A continuación podremos ver cómo los sentimientos y las pasiones humanas, expuestas por Hobbes y Hume como fundamento de la moral, han sido retomados en las teorías contemporáneas que explican la dimensión moral a partir del desarrollo socio afectivo.

\section{Desarrollo moral y emoción}

$\mathrm{Al}$ observar las tendencias recientes en el campo de la psicología del desarrollo moral, llama la atención aquella que defiende el vínculo entre el desarrollo socioemocional y el entendimiento y cumplimiento de normas o principios morales. Eisenberg (2000), Kochanska (1997) y Hoffman $(1983,1984,2002)$, entre otros autores, han investigado en torno al rol que cumplen emociones como la vergüenza, la culpa o la empatía en la internalización de normas y la motivación de la conducta prosocial. A partir de las evidencias recogidas han podido defender la idea de que sólo en la medida en que el ser humano experimenta cierto tipo de emociones complejas, es capaz de actuar conforme a principios morales.

Si bien es cierto que otras perspectivas del desarrollo también han reconocido el papel que cumplen las emociones en la moral, lo que caracteriza esta propuesta es el hecho de considerar que aquello que impide actuar a favor o en contra de ciertos principios es la experien- cia de una emoción desagradable o agradable que acompaña el acto. Con base en la idea de la existencia de emociones morales como la empatía que impulsan la acción moral, esta perspectiva pretende explicar la acción humana en cinco tipo de encuentros o dilemas morales: la del espectador inocente del daño que otro sufre; la del transgresor que causa daño; la del transgresor virtual que siendo inocente cree que causa daño; la de lección de varios interpelantes morales, en donde se presenta el dilema de elegir beneficiar a unos, mientras se puede dañar a otros; y la de atención de justicia, que se muestra como una situación similar a la anterior, pero donde intervienen aspectos como el conflicto de intereses y cuestiones abstractas como los derechos humanos, el deber y la reciprocidad (Hoffman, 2002). Todas las situaciones tienen como motor la empatía en tanto que tipo de emoción moral.

Eisenberg (2000) explica la existencia de emociones morales y no morales. Las primeras ocupan un papel fundamental en la vida moral: no sólo ponen un límite a los actos que pueden ocasionar un daño directo o indirecto a otras personas, también motivan actos en beneficio de otros. Por su parte, las emociones no morales, como los estados de humor (mood states), se relacionan con la percepción y el juicio acerca de lo justo o lo injusto.

La pena (embarrasment), la culpa (guilt) y la vergüenza (shame) son llamadas emociones morales de auto-conciencia. Han sido etiquetadas de esta forma porque dependen de un entendimiento de la individualidad y de la evaluación del yo. Son emociones negativas que evitan la trasgresión de las normas establecidas.

De estas tres emociones, la pena es la emoción menos intensa y negativa y la más fugaz. Conlleva a una crítica de menor grado hacia el yo. Puede comprenderse como el simple bochorno experimentado tras haber infringido una convención social.

Por otra parte, la culpa ha sido definida de diversas maneras. Para Eisenberg (2000), desde 
la perspectiva del desarrollo moral, la definición de la culpa debe distinguirse de la concepción mantenida por la teoría psicoanalítica. Freud (1929 [1930]; citado por Hoffman, 1983) concibió la culpa como el sentimiento que surge ante el conflicto entre la combinación de erotismo y hostilidad dirigida hacía los padres y la ansiedad por la posibilidad de la pérdida del amor parental. En este sentido, la culpa se relaciona con el sufrimiento y los problemas de adaptación. En contraste, Eisenberg (2000) propone que la idea de culpa que mejor se acomoda al concepto de emoción moral se refiere más al arrepentimiento o remordimiento que acompaña una acción inadecuada. Al sentir culpa, el infractor acepta su responsabilidad y busca enmendar el daño o recibir un castigo.

Aunque la vergüenza y la culpa tienden a confundirse, la diferencia entre ambas se encuentra en que la primera de ellas implica una mayor intensidad y una crítica más severa al yo. Quien se avergüenza por haber traspasado un límite permitido, se evalúa de manera negativa, siente desprecio por sí mismo y busca evitar u ocultarse de los demás (Ferguson \& Stegge, 1998; citado por Eisenberg, 2000).

De otro lado, la repuesta de empatíaidentificación (empathy-related) es considerada también como una emoción moral (Eisenberg, 2000; Hoffman, 1984). Ésta es definida como una respuesta afectiva que proviene de la aprehensión o comprensión de las condiciones o estados emocionales experimentados por otros; la respuesta afectiva empática guarda una similitud aquello que la otra persona siente o puede llegar a sentir en una situación particular. Hoffman (2002) la describe como un estado afectivo unido a un principio moral, el cual se activa en situaciones particulares con víctimas. La función de este afecto es motivar el juicio o la acción ante dichas circunstancias, motivación que no genera de manera suficiente el principio moral por sí solo.

La empatía ha sido relacionada con la regulación emocional y las diferencias disposi- cionales de la personalidad y el temperamento. Es similar a otra emoción moral llamada simpatía (sympathy), pero aunque ésta última es una respuesta emocional que se deriva igualmente a partir de la comprensión de los estados emocionales o condiciones experimentados por otros, no supone sentir lo que el otro siente sino más bien experimentar dolor o preocupación por la situación que experimenta el otro. Por ende, la simpatía está más comprometida con el altruismo y la conducta prosocial que la empatía-identificación.

El segundo aspecto del que se ocupa la teoría socio emocional es la internalización de los principios o las normas morales. Hoffman (1983) define una norma moral como aquella que ordena actuar, tomando en consideración las necesidades de otros de la misma manera en que se toman en consideración las propias necesidades. Esto supone actuar en búsqueda del bienestar propio y de los otros, al tiempo que se evita causar cualquier tipo de daño. Se considera que una norma moral ha sido internalizada cuando la persona siente la obligación de actuar conforme a la norma, aun en la ausencia de cualquier posibilidad de castigo o recompensa externa. Antes de la internalización de la norma, el niño actúa con el fin de mantener la armonía en la relación con sus padres y evitar el castigo o la pérdida de amor.

La internalización conlleva, además, un conflicto entre la tentación de actuar en beneficio propio, de manera egoísta, y actuar conforme a las necesidades de otros. Las emociones morales aparecerán en beneficio del cumplimiento de la norma. Visto de otra forma, se hace necesaria una experiencia significativa que nos lleve a abandonar los beneficios del comportamiento egoísta. Cuando hablamos de internalización de la norma suponemos que dicha experiencia debe darse al interior del individuo y corresponde a los estados emocionales morales. El individuo actuará, entonces, eludiendo experimentar las emociones morales que le resultan desagradables: se evita dañar a otros para no 
sentir culpa o la vergüenza y ayudará a otros, en tanto se identifica con las emociones que ello experimentan tras su sufrimiento.

Finalmente, al referirse a la internalización, la teoría socio emocional se presenta como una propuesta actual que recoge aspectos planteados por la teoría psicoanalítica, la teoría del aprendizaje social y la teoría de la atribución. Para las tres teorías, desde el exterior se genera la prohibición, el castigo o la presión con respecto a ciertos actos, lo cual le permite al niño comprender cuáles son los principios a los que debe ajustar su conducta: La teoría psicoanalítica plantea que el conflicto entre el deseo y la cultura hace emerger la culpa, emoción que da lugar a la formación de la instancia denominada súper yo, que es la instancia moral o el conjunto de normas culturales internalizadas, distinta al ello (lo inconsciente) y el yo (lo consciente) (Freud, 1929 [1930]); la teoría del aprendizaje social propone que el niño asocia el castigo y la acción, de tal manera que luego es capaz de anticipar la aparición de la sanción y como efecto experimenta un estado de ansiedad que le impide actuar (Eisenberg, 2000). Para la teoría de la atribución, la emoción emerge gracias al significado que el niño le otorga al castigo que espera recibir por una acción incorrecta o a los posibles efectos dañinos de la acción cometida (Eisenberg, 2000).

A partir de allí, Eisenberg (2000) y Hoffman (1983) le otorgan importancia a la disciplina impartida por los padres o cuidadores del niño. Plantean que la importancia de la disciplina aparece ante el conflicto entre los requerimientos morales de una situación determinada y el deseo de satisfacción de los deseos propios. Las sanciones y recompensas externas conducen al cumplimiento de la norma. La internalización de la norma supone el reemplazo del premio o el castigo por una emoción agradable o desagradable en el niño. En conclusión, la norma internalizada motiva el acto moral en función de la búsqueda o evitación de ciertos estados emocionales.

\section{Conclusiones}

La filosofía moral se ha caracterizado por su interés en la búsqueda de argumentos que permitan explicar los fundamentos de los sistemas éticos y políticos. De esta manera, el emotivismo moral funciona en la filosofía como una teoría del significado de los conceptos morales que argumento que dichos fundamentos se encuentran sujetos a nuestros afectos o sentimientos. Mientras tanto, la psicología moral se ha interesado por explicar qué tipo de factores influyen en la forma en que elaboramos juicios morales y actuamos frente a ciertas situaciones que comprometen un daño hacia los demás. Así, la diferencia entre ambas disciplinas queda establecida por la diferencia entre la dimensión normativa y descriptiva de la moral.

No obstante, hemos visto cómo las reflexiones propias de la tradición filosófica traspasan la frontera disciplinar y son retomadas desde un abordaje científico. Este es el caso de las teorías contemporáneas del desarrollo moral que defienden la influencia de los afectos en nuestros actos y juicios morales. Es evidente que el interés de los abordajes teóricos presentados en la psicología no ha tenido un fuerte interés en el problema del significado del lenguaje moral. Por lo tanto, no podemos referirnos a un emotivismo con la acepción que ha tenido en filosofía. Lo que no nos impide resaltar las coincidencias entre las posturas filosóficas y psicológicas descritas, permitiéndonos hablar de una forma de emotivismo en Psicología o la postura que defiende el componente afectivo como fundamento de nuestra moralidad.

La anterior idea nos sugiere que se nos muestra una misma postura desde dos niveles de discusión distintos frente al mismo problema, el de la moral. Empero, el intento de la tradición empirista por construir una ciencia del hombre sobre una base científica, aspecto que en el que avanza mucho más Hume que Hobbes y que queda claro por las cualidades del Tratado de la naturaleza bumana, puede considerarse como un 
antecedente de suma importancia, quizás poco reconocido para la psicología moral. Una lectura más atenta de estas discusiones en la filosofía moral puede generar reflexiones aún más interesantes para el abordaje empírico.

Finalmente, cabe aclarar que lo expuesto en este documento representa sólo un parte de las temáticas y posturas que protagonizan el debate sobre la moral. Aunque se dejan de lado críticas a este enfoque y discusiones con otras posturas, consideramos que la información suministrada puede ser de gran utilidad para aquellos interesados en la cuestión moral desde sus diversas disciplinas. 


\section{Referencias bibliográficas}

Hobbes, T. (2010). Leviatán o la forma y poder de una república Eclesiástica y Civil. Bogotá: Skla.

Hoffman, M. (1983). Affective and cognitive processes in moral internalization. En E. Higgins, D. Ruble \& W.Hartup (Eds.), Social Cognition and Social Development: $A$ sociocultural Perspective (pp. 236-77). Cambridge University Press.

(1984). Emphaty, Its Limitations, and Its Role in a Comprehensive Moral Theory. En W. Kurtines \& S. Gewirtz (Eds.) Morality, Morla behavior, and Moral Development (pp. 283- 330). Wiley.

. (2002). Desarrollo, moral y empatía. Barcelona: Idea Books Universitaria.

Hume, D. (2005). Tratado de la naturaleza Humana. México D. F.: Porrúa.

Kochanska, G. (1997). Multiple Pathways to Conscience for Children With Differente Temperaments: From Toddlerhood to Age 5. Development Psychology, 333(2), pp. 228-240.

Eisenberg, N. (2000). Emotion, Regulation, and Moral Development. Annual Reviews Psychology, 51, pp. 665-697.
Freud, S. (1929 [1930]). El malestar en la Cultura. Madrid: Amorrortu.

Harman, G. (1996). La Naturaleza de la Moralidad: una Introducción a la ética. México D.F.: UNAM.

McIntyre, A. (2001). Tras la Virtud. Barcelona: Crítica.

- (2006). Historia de la ética.

Barcelona: Paidos.

Rachels, J. (2009). Introducción a la filosofía Moral. México D. F.: FCE.

Schneewind, J. B. (2009). La Invención de la Autonomia: una Historia de la Filosofía Moral moderna. México D. F.: FCE.

Strauss , L., \& Cropsey, J. (1996). Historia de la filosofía politica. México D. F.: FCE.

Yáñez. J., Perdomo, A., \& Mojica, A. (s/f). La moral, la psicología del desarrollo y la teoría de los dominios. Documento grupo de investigación Desarrollo Moral y Justicia. Bogotá: Universidad Nacional de Colombia. 